\title{
FREDHOLM WEIGHTED COMPOSITION OPERATORS
}

\author{
ChIng-On Lo AND Anthony WAI-KEUng LoH
}

Abstract. We show that Fredholm weighted composition operators on $L^{p}$-spaces with nonatomic measures are precisely the invertible ones. We also characterize the classes of Fredholm and invertible weighted composition operators on $l^{p}$. Furthermore, the closedness of ranges and Fredholmness of these operators on $H^{p}$-spaces of the unit disk are investigated.

Mathematics subject classification (2010): 47B33. operators.

Keywords and phrases: Weighted composition operators, Lebesgue spaces, Hardy spaces, Fredholm

\section{REFERENCES}

[1] P. S. Bourdon, Fredholm multiplication and composition operators on the Hardy space, Integral Equations Operator Theory 13 (1990), 607-610.

[2] J. A. Cima, J. Thomson AND W. Wogen, On some properties of composition operators, Indiana Univ. Math. J. 24 (1974), 215-220.

[3] M. D. Contreras and A. G. Hernández-Díaz, Weighted composition operators on Hardy spaces, J. Math. Anal. Appl. 263 (2001), 224-233.

[4] G. Gunatillake, Invertible weighted composition operators, J. Funct. Anal. 261 (2011), 831-860.

[5] D. K. Gupta AND B. S. KomaL, Fredholm composition operators on weighted sequence spaces, Indian J. Pure Appl. Math. 14 (1983), 293-296.

[6] D. J. Harrington, Co-rank of a composition operator, Canad. Math. Bull. 29 (1986), 33-36.

[7] M. R. JABBARZADEH, Weighted composition operators between $L^{p}$-spaces, Bull. Korean Math. Soc. 42 (2005), 369-378.

[8] P. LefÈvre, D. Li, H. QuefFélec AND L. RodrígueZ-PiazZA, Some revisited results about composition operators on Hardy spaces, Rev. Mat. Iberoam. 28 (2012), 57-76.

[9] C. O. Lo, Weighted composition operators between $L^{p}$-spaces, Thesis, The University of Hong Kong, 2002.

[10] B. D. MACCLUER, Compact composition operators on $H^{p}\left(B_{N}\right)$, Michigan Math. J. 32 (1985), 237 248.

[11] S. OhNo AND H. TAKAGI, Some properties of weighted composition operators on algebras of analytic functions, J. Nonlinear Convex Anal. 2 (2001), 369-380.

[12] R. K. Singh And A. Kumar, Characterizations of invertible, unitary, and normal composition operators, Bull. Austral. Math. Soc. 19 (1978), 81-95.

[13] R. K. Singh AND T. Veluchamy, Atomic measure spaces and essentially normal composition operators, Bull. Austral. Math. Soc. 27 (1983), 259-267.

[14] R. K. Singh And T. Veluchamy, Nonatomic measure spaces and Fredholm composition operators, Acta Sci. Math. (Szeged) 51 (1987), 461-465.

[15] H. TAKAGI, Fredholm weighted composition operators, Integral Equations Operator Theory 16 (1993), 267-276.

[16] L. K. ZhaO, Fredholm weighted composition operators on Hardy space, J. Math. Res. Appl. 33 (2013), 361-364. 